TRANSACTIONS OF THE

AMERICAN MATHEMATICAL SOCIETY

Volume 353, Number 5, Pages 1985-1993

S 0002-9947(01)02752-0

Article electronically published on January 10, 2001

\title{
AHIEZER-KAC TYPE FREDHOLM DETERMINANT ASYMPTOTICS FOR CONVOLUTION OPERATORS WITH RATIONAL SYMBOLS
}

\author{
SERGIO ALBEVERIO AND KONSTANTIN A. MAKAROV
}

\begin{abstract}
Fredholm determinant asymptotics of convolution operators on large finite intervals with rational symbols having real zeros are studied. The explicit asymptotic formulae obtained can be considered as a direct extension of the Ahiezer-Kac formula to symbols with real zeros.
\end{abstract}

\section{INTRODUCTION}

The continuous version of the Szegö limit theorem [1, 18], [19] gives a two-term asymptotics for the Fredholm determinants of convolution operators on $L^{2}(0, \tau)$ as $\tau \rightarrow \infty$. Under suitable conditions on the kernel function $k(t)$ the Fredholm determinant of the integral operator $I-K_{\tau}$, with $K_{\tau}$ defined by

$$
K_{\tau} \varphi(t)=\int_{0}^{\tau} k(t-s) \varphi(s) d s,
$$

is asymptotically given as $\tau \rightarrow+\infty$ by the Ahiezer-Kac formula

$$
\operatorname{det}\left(I-K_{\tau}\right)=\exp \left(f(0) \tau+\int_{0}^{\infty} z f(z) f(-z) d z+o(1)\right),
$$

where the function

$$
f(z)=\frac{1}{2 \pi} \int_{\mathbb{R}} e^{-i x z} \log (W(x)) d x, \quad z \in \mathbb{R},
$$

is just the Fourier transform of the logarithm of the symbol $W$ of the integral operator $I-K_{\tau}$,

$$
W(x)=1-\int_{\mathbb{R}} e^{-i x s} k(s) d s, \quad x \in \mathbb{R} .
$$

This is so provided that the symbol $W$

- satisfies some smoothness conditions,

- attains its limit values at infinity rather fast,

- does not vanish on the real axis, and

- has zero winding number (the index) with respect to the real axis.

The latter property is usually called "zero index condition".

In the case where $W$ has real zeros the Fredholm determinant $\operatorname{det}\left(I-K_{\tau}\right)$, considered as a function of $\tau$, might have an infinite series of zeros, and hence the asymptotic representation (1.1) is not valid in general.

Received by the editors October 17, 1997.

1991 Mathematics Subject Classification. Primary 45P05, 47B35; Secondary 47A68, 47G10. 
The study of the case where the symbol is vanishing and/or the zero index condition is violated has been undertaken (for Toeplitz operators) by Fisher and Hartwig 12], 13] and continued by Widom [20], Basor [6], and Böttcher and Silbermann [8], [9], who showed that the asymptotics may be oscillatory in nature (see also [10] for further references).

We concentrate our attention on the case of convolution operators with rational symbols that may have real zeros.

The case of rational symbols is well understood. In [15] (see also [10]) one can find an exhaustive exposition of results in the theory of convolution type operators with rational symbols on a finite interval, including rather explicit representations for resolvents, eigenfunctions, Fredholm determinants, etc. In particular, an explicit representation for 2-modified Fredholm determinants, known as a Wiener-Hopf analog of Day's formula [1], holds.

Day's Formula ([7]). Let the symbol $W$ of the integral operator $\left(I-K_{\tau}\right)$ be a rational function of the form

$$
W(x)=\frac{\prod_{n=1}^{q+s}\left(x-\xi_{n}\right)}{\prod_{l=1}^{q}\left(x+i \lambda_{l}\right) \prod_{m=1}^{s}\left(x-i \mu_{m}\right)},
$$

where $q \geq 1, s \geq 1$,

$$
\operatorname{Re}\left(\lambda_{l}\right)>0, l=1, \ldots, q, \quad \operatorname{Re}\left(\mu_{m}\right)>0, m=1, \ldots, s,
$$

and $\xi_{1}, \ldots, \xi_{q+s}$ are pairwise distinct complex numbers. Then for all $\tau \in(0, \infty)$

$$
\operatorname{det}_{2}\left(I-K_{\tau}\right)=\exp \left(\tau\left(k(0+0)-\sum_{l \in Q} \lambda_{l}\right)\right) \sum_{M} W_{M} e^{w_{M} \tau},
$$

where det $_{2}$ denotes the 2-modified Fredholm determinant. Here the sums are taken over the sets $Q=\{1, \ldots, q\}$ and $M \subset\{1,2, \ldots, q+s\}$ with $|M|=s$. Letting $\bar{M}=$ $\{1, \ldots, q+s\} \backslash M$ and $S=\{1, \ldots, s\}$, the constants $W_{M}$ and $w_{M}$ can be given by

$$
\begin{gathered}
W_{M}=\frac{\prod_{l \in Q, k \in M}\left(\lambda_{l}-i \xi_{k}\right) \prod_{j \in \bar{M}, m \in S}\left(i \xi_{j}+\mu_{m}\right)}{\prod_{l \in Q, m \in S}\left(\lambda_{l}+\mu_{m}\right) \prod_{j \in \bar{M}, k \in M}\left(i \xi_{j}-i \xi_{k}\right)}, \\
w_{M}=\sum_{j \in \bar{M}} i \xi_{j} .
\end{gathered}
$$

If $q=0$ or $s=0$, the operator $\left(I-K_{\tau}\right)$ is of Volterra type and therefore $\operatorname{det}_{2}\left(I-K_{\tau}\right)=1$ for all $\tau \in(0, \infty)$.

In spite of the fact that Day's formula gives an explicit representation of the Fredholm determinants for the truncated Wiener-Hopf operators $\left(I-K_{\tau}\right)$ in terms of zeros and poles of the symbol, we have not found in the literature asymptotic representations which can be considered as direct extensions of the Ahiezer-Kac formula to the case of symbols with real zeros. We mean such generalizations which

- on the one hand provide asymptotic formulae in terms of integral characteristics of the symbol, and

- on the other hand might be generalized to the case of non-rational (say, meromorphic) symbols. (Such convolution operators with meromorphic symbols arise in particular in our study of the universality of the Efimov effect [2], [3], [4], [5]; see also [14].) 
In the present paper we introduce a new class of symbols (with real zeros) that satisfy the generalized zero index condition (see definitions 2.1 2.2), and we get a natural direct extension of the Ahiezer-Kac formula (Theorem [2.3). This extension describes determinant asymptotics in terms of integral characteristics of the symbol like the Fourier transform of the logarithm of the symbol in the original AhiezerKac formula (1.1) and, moreover, takes into account the oscillatory character of the asymptotics.

\section{Generalized Zero index CONDition And DEterminant ASYMPtotics}

Let $K_{\tau}$ be a convolution integral operator on $L^{2}(0, \tau), \tau>0$, with rational symbol $W$ of the form (1.3), having $s \geq 1$ simple poles $\left\{i \mu_{l}\right\}_{m=1}^{s}$ in the open upper half-plane, $q \geq 1$ simple poles $\left\{-i \lambda_{l}\right\}_{l=1}^{q}$ in the open lower half-plane, and $q+s$ simple zeros. For definiteness we assume that the symbol has $u$ zeros in the open upper half-plane, $\ell$ zeros in the open lower half-plane and, therefore, it has exactly $q+s-(u+\ell) \geq 0$ zeros on the real axis.

Definition 2.1. The difference between the number of poles and the number of zeros of the symbol $W$ in the open upper half-plane

$$
n_{+}(W)=s-u
$$

resp. in the open lower half-plane

$$
n_{-}(W)=q-\ell
$$

is called the deficiency index of the symbol in the upper (resp. lower) half-plane.

Definition 2.2. We shall say that a rational symbol $W$ satisfies the generalized zero index condition if its deficiency indices $n_{ \pm}(W)$ are both nonnegative integers.

It is easy to see that if a rational symbol $W$ without real zeros has zero winding number (with respect to the real axis), then the number of its poles in the upper half-plane coincides with the number of the zeros there and hence the symbol $W$ has deficiency indices $(0,0)$. Thus the generalized zero index condition is a natural extension of the usual "zero index condition" to the case of rational symbols with real zeros.

Let us also note that every rational symbol $W$ satisfying the generalized zero index condition can be obtained as the pointwise limit of a sequence of rational symbols (without real zeros) of index zero.

Suppose that the symbol $W$ has $r(r=q+s-(u+\ell))$ simple real zeros $\xi_{i_{1}, \ldots,} \xi_{i_{r}}$, that is,

$$
W\left(\xi_{i_{k}}\right)=0 \quad \text { and } \quad W^{\prime}\left(\xi_{i_{k}}\right) \neq 0, \quad k=1, \ldots, r .
$$

Let $\Gamma_{\delta}$ be a contour going from $-\infty$ to $+\infty$ along the real axis outside some small neighborhood of the real zeros $\xi_{i_{1}}, \ldots, \xi_{i_{r}}$, passing around each zero $\xi_{i_{k}}, k=1, \ldots, r$, along a semi-circle of radius $\delta$ either in the counterclockwise direction (in the lower half-plane from below) or in the clockwise one (in the upper half-plane from above).

For $\delta$ small enough every such contour $\Gamma_{\delta}$ contains neither the poles nor the zeros of the symbol $W$ and, therefore, the winding number (the index) of the symbol with respect to the contour $\Gamma_{\delta}$ is well defined. Note that this index does not depend on $\delta$ if the family of contours $\left\{\Gamma_{\delta}\right\}_{\delta}$ belongs to the same homotopy class $\gamma$ on the complex plane $\mathbf{C} \backslash\left\{\xi_{i_{1}}, \ldots, \xi_{i_{r}}\right\}$ without points $\xi_{i_{1}}, \ldots, \xi_{i_{r}}$. It is also clear that the index of the symbol $W$ with respect to such contours depends in fact on the corresponding 
homotopy class $\gamma$ only, not on the concrete representative $\Gamma_{\delta} \in \gamma$ from this class (for $\delta$ small enough, of course).

We shall call this index the index of the homotopy class $\gamma$.

Let us denote by $\pi(W)$ the set of homotopy classes described above having zero index,

$$
\pi(W)=\{\gamma \mid \operatorname{ind}(\gamma)=0\} .
$$

It is easy to understand that the symbol satisfies the generalized zero index condition if and only if the set $\pi(W)$ is not empty, that is, there is at least one homotopy class $\gamma$ with zero index ind $(\gamma)=0$. In this case for the symbol $W$ with deficiency indices $\left(n_{+}(W), n_{-}(W)\right)$ we have $\left(\begin{array}{c}n_{+}(W)+n_{-}(W) \\ n_{+}(W)\end{array}\right)$ different homotopy classes of zero index, that is,

$$
|\pi(W)|=\left(\begin{array}{c}
n_{+}(W)+n_{-}(W) \\
n_{+}(W)
\end{array}\right) .
$$

Assume that the symbol $W$ satisfies the generalized zero index condition and, in addition, the following condition holds:

$$
W-1 \in L^{1}(\mathbb{R}) .
$$

For every homotopy class $\gamma$ of zero index, $\gamma \in \pi(W)$, introduce the Fourier transform $f_{\gamma}(z)$ of the logarithm of the symbol associated with $\gamma$ by the following contour integral:

$$
f_{\gamma}(z)=\frac{1}{2 \pi} \int_{\Gamma_{\delta}} e^{-i x z} \log (W(x)) d x, \quad \Gamma_{\delta} \in \gamma, \quad \gamma \in \pi(W)
$$

The function $f_{\gamma}(z)$ is obviously a well defined continuous function in $z$. Moreover, $f_{\gamma}(z)$ depends on the homotopy class $\gamma \in \pi(W)$ only, not on the particular choice of the contour $\Gamma_{\delta} \in \gamma$.

Now we are prepared for the formulation of the central result of the paper.

Theorem 2.3. Let $K_{\tau}$ be a convolution integral operator on $L^{2}(0, \tau)$ with rational symbol $W$ given by (1.3). Suppose that $W$ satisfies condition (2.3). Then $K_{\tau}$ is of trace class and, therefore, the Fredholm determinant $\operatorname{det}\left(I-K_{\tau}\right)$ is well defined.

If, in addition, the symbol $W$ satisfies the generalized zero index condition, then we have the following asymptotic representation as $\tau \rightarrow \infty$ :

$$
\operatorname{det}\left(I-K_{\tau}\right)=\sum_{\gamma: \operatorname{ind}(\gamma)=0} \exp \left(f_{\gamma}(0) \tau+\lim _{\varepsilon \rightarrow+0} \int_{0}^{\infty} z f_{\gamma}(z) f_{\gamma}(-z) e^{-\varepsilon z} d z\right)+\theta(\tau),
$$

where $f_{\gamma}(z)$ given by (2.4) denotes the Fourier transform of the logarithm of the symbol associated with the homotopy class $\gamma \in \pi(W)$. Every summand in (2.5) has the same exponential order $\omega$,

$$
\omega=\operatorname{Re}\left(f_{\gamma}(0)\right), \quad \gamma \in \pi(W),
$$

and the remainder $\theta(\tau)$ is of the form

$$
\theta(\tau)=o\left(e^{\omega \tau}\right), \quad \tau \rightarrow \infty .
$$


Proof. Under condition (2.3) the Fourier transform $\widehat{k}$ of the kernel function $k$ is a bounded integrable function

$$
\widehat{k} \in L^{1}(\mathbb{R}) \cap L^{\infty}(\mathbb{R}) .
$$

By Mercer's theorem, (2.7) implies that the integral operator $K_{\tau}$ is a trace class operator (see, e.g., [16]). Hence the Fredholm determinant $\operatorname{det}\left(I-K_{\tau}\right.$ ) is well defined and

$$
\operatorname{det}\left(I-K_{\tau}\right)=e^{-\operatorname{tr}\left(K_{\tau}\right)} \operatorname{det}_{2}\left(I-K_{\tau}\right),
$$

where det $_{2}$ denotes the 2-modified Fredholm determinant. Condition (2.7) in particular means that the kernel $k(t)$ is a continuous function, and hence

$$
\operatorname{tr}\left(K_{\tau}\right)=\tau k(0) .
$$

Using (2.8) and (2.9), Day's formula (11.5) can be read as follows:

$$
\operatorname{det}\left(I-K_{\tau}\right)=\exp \left(-\tau \sum_{l \in Q} \lambda_{l}\right) \cdot \sum_{M} W_{M} e^{w_{M} \tau},
$$

where the coefficients $W_{M}$ and the exponents $w_{M}$ are given by (1.61) and (1.7) respectively.

The computation of the leading terms of the asymptotics as $\tau \rightarrow \infty$ for the Fredholm determinant (2.10) amounts to the solution of a purely combinatorial problem which by (1.7) is reduced to the search of $s$-element subsets $M, M \subset$ $\{1,2, \ldots, q+s\}$, giving a maximum $w$ for the real part of the exponents $w_{M}$ :

$$
w=\max _{M}\left(w_{M}\right)=\max _{M}\left(\operatorname{Re}\left(\sum_{j \in \bar{M}} i \xi_{j}\right)\right) .
$$

Recall that the symbol $W$ has $s$ poles and $u$ zeros in the upper half-plane, $q$ poles and $\ell$ zeros in the lower half-plane, and hence $W$ has exactly $r=q+s-(u+\ell) \geq 0$ zeros on the real axis. Denote by $U, L$, and $R$ the corresponding index sets enumerating the zeros of $W$.

Clearly the maximum (2.11) coincides with the real part of the sum of all zeros of the symbol $W$ from the lower half-plane, that is,

$$
w=\operatorname{Re}\left(\sum_{\ell \in L} i \xi_{\ell}\right)
$$

Therefore, the "extremal" index subsets $M$ are of the form

$$
M=\{1, \ldots, q+s\} \backslash(L \cup Z), \quad|M|=s,
$$

where $Z$ runs over all $(q-\ell)$-element subsets of the index set $R$ :

$$
Z \subset R, \quad|Z|=q-\ell=n_{-}(W) .
$$

It is also clear that there are $\left(\begin{array}{c}r \\ q-\ell\end{array}\right)$ subsets $M$ such that the corresponding exponents $w_{M}$ solve the extremum problem (2.11). The total number of these subsets can also be expressed in terms of the deficiency indices $n_{ \pm}(W)$ of the symbol $W$ given by (2.1) and (2.2):

$$
\left(\begin{array}{c}
r \\
q-\ell
\end{array}\right)=\left(\begin{array}{c}
n_{+}(W)+n_{-}(W) \\
n_{-}(W)
\end{array}\right)=\left(\begin{array}{c}
n_{+}(W)+n_{-}(W) \\
n_{+}(W)
\end{array}\right) .
$$


With every "extremal" set $M$ of the form (2.13) we can associate a homotopy class $\gamma$ of contours $\Gamma_{\delta}$ going around the real zeros $\left\{\xi_{\ell}\right\}_{\ell \in Z}$ in the clockwise direction and surrounding all the other real zeros $\left\{\xi_{u}\right\}_{u \in R \backslash Z}$ in the counterclockwise direction, as it was described above.

Roughly speaking, the contour $\Gamma_{\delta}$, or more generally the corresponding homotopy class $\gamma$, determined by the contour $\Gamma_{\delta} \in \gamma$, assigns $n_{+}(W)$ real zeros of the symbol to the upper half-plane and the remaining $n_{-}(W)$ real zeros to the lower one. Therefore, every such contour associated with any extremal set $M$ divides the singularities (zeros and poles) of the symbol $W$ in such a manner that the total number of poles of $W$ from above with respect to the contour coincides with the total number of zeros there, and therefore the corresponding homotopy class $\gamma$ has index zero, i.e., $\gamma \in \pi(W)$.

Conversely, every homotopy class $\gamma$ of zero index, $\gamma \in \pi(W)$, determines in a unique manner an "extremal" set $M$ of the type (2.13) such that the corresponding coefficient $w_{M}$ solves the extremum problem (2.11). Therefore, we have a one-toone correspondence

$$
\gamma \longmapsto M_{\gamma}
$$

between all homotopy classes of zero index on the one hand, and all sets (2.13), on the other.

Using the correspondence (2.14) we can extract from (2.10) the leading terms of the Fredholm determinant asymptotics as follows:

$$
\operatorname{det}\left(I-K_{\tau}\right)=\sum_{\gamma \in \pi(W)} W_{M_{\gamma}} \exp \left(\tau\left(w_{M_{\gamma}}-\sum_{l \in Q} \lambda_{l}\right)\right)+\theta(\tau),
$$

where

$$
\theta(\tau)=o\left(e^{\omega \tau}\right)
$$

with

$$
\omega=\operatorname{Re}\left(w_{M_{\gamma}}-\sum_{l \in Q} \lambda_{l}\right)=w-\operatorname{Re}\left(\sum_{l \in Q} \lambda_{l}\right)
$$

and $w$ given by (2.11).

Given a homotopy class $\gamma \in \pi(W)$, let us compute the corresponding Fourier transform $f_{\gamma}(z)$ (2.4) associated with the class $\gamma$.

Let $\Gamma_{\delta}$ be a contour from the class $\gamma$. Under condition (2.3) we can integrate by parts in (2.4) to get

$$
f_{\gamma}(z)=\frac{1}{2 \pi i z} \int_{\Gamma_{\delta}} e^{-i x z} \frac{W(x)^{\prime}}{W(x)} d x, \quad \gamma \in \pi(W) .
$$

Note that by definition of the "extremal" index set $M_{\gamma}$ all the zeros $\left\{\xi_{j}\right\}_{j \in \overline{M_{\gamma}}}$ are situated from below the contour $\Gamma_{\delta}$. In particular,

$$
\operatorname{Im}\left(\xi_{j}\right) \leq 0, \quad j \in \overline{M_{\gamma}},
$$

and

$$
\operatorname{Im}\left(\xi_{k}\right) \geq 0, \quad k \in M_{\gamma}
$$


Using now the theorem of residua for $z>0$ (closing the contour of integration $\Gamma_{\delta}$ in the lower half-plane), we get

$$
f_{\gamma}(z)=\frac{1}{z}\left(\sum_{l \in Q} e^{-\lambda_{l} z}-\sum_{j \in \overline{M_{\gamma}}} e^{-i \xi_{j} z}\right), \quad z>0 .
$$

Analogously, for $z<0$, closing the contour $\Gamma_{\delta}$ to the upper half-plane, we obtain

$$
f_{\gamma}(z)=-\frac{1}{z}\left(\sum_{m \in S} e^{\mu_{m} z}-\sum_{k \in M_{\gamma}} e^{-i \xi_{k} z}\right), \quad z<0 .
$$

In particular, by (2.19) for $f_{\gamma}(0)$ we have the expression

$$
f_{\gamma}(0)=\sum_{j \in \overline{M_{\gamma}}} i \xi_{j}-\sum_{l \in Q} \lambda_{l}=w_{M_{\gamma}}-\sum_{l \in Q} \lambda_{l}
$$

Comparing (2.21) with (2.15), we can rewrite the asymptotics (2.15) as follows:

$$
\operatorname{det}\left(I-K_{\tau}\right)=\sum_{\gamma \in \pi(W)} W_{M_{\gamma}} e^{\tau f_{\gamma}(0)}+\theta(\tau),
$$

where the error term is of the form (2.16) and, in addition, (2.6) holds. Hence, in order to complete the proof of the theorem we have to establish a series of equalities

$$
W_{M_{\gamma}}=\exp \left(\lim _{\varepsilon \rightarrow+0} \int_{0}^{\infty} z f_{\gamma}(z) f_{\gamma}(-z) e^{-\varepsilon z} d z\right), \quad \gamma \in \pi(W) .
$$

In fact, this result is hidden in the Ahiezer-Kac formula for symbols without real zeros combined with Day's formula. We prefer, however, to give a direct proof of (2.22).

Given $\gamma \in \pi(W)$, by (2.19) and (2.20) for $z>0$ we have

$$
\begin{aligned}
z f_{\gamma}(z) f_{\gamma}(-z) & =\frac{1}{z}\left(\sum_{l \in Q} e^{-\lambda_{l} z}-\sum_{j \in \overline{M_{\gamma}}} e^{-i \xi_{j} z}\right)\left(\sum_{m \in S} e^{-\mu_{m} z}-\sum_{k \in M_{\gamma}} e^{i \xi_{k} z}\right) \\
& =\frac{1}{z}\left(\sum_{l \in Q, m \in S} e^{-\left(\lambda_{l}+\mu_{m}\right) z}-\sum_{l \in Q, k \in M_{\gamma}} e^{-\left(\lambda_{l}-i \xi_{k}\right) z}\right) \\
& +\frac{1}{z}\left(\sum_{j \in \overline{M_{\gamma}}, k \in M_{\gamma}} e^{-\left(i \xi_{j}-i \xi_{k}\right) z}-\sum_{j \in \overline{M_{\gamma}}, m \in S} e^{-\left(i \xi_{k}+\mu_{m}\right) z}\right) .
\end{aligned}
$$

By (1.4), (2.17), and (2.18) one obtains the series of inequalities

$$
\begin{aligned}
& \operatorname{Re}\left(\lambda_{l}+\mu_{m}\right)>0, \quad l \in Q, \quad m \in S, \\
& \operatorname{Re}\left(\lambda_{l}-i \xi_{k}\right)>0, \quad l \in Q, \quad k \in M_{\gamma}, \\
& \operatorname{Re}\left(i \xi_{j}-i \xi_{k}\right) \geq 0, \quad j \in \overline{M_{\gamma}}, \quad k \in M_{\gamma}, \\
& \operatorname{Re}\left(i \xi_{j}+\mu_{k}\right)>0, \quad j \in \overline{M_{\gamma}}, \quad m \in S .
\end{aligned}
$$

Therefore, every summand in (2.23) is a bounded function and, in particular,

$$
z f_{\gamma}(z) f_{\gamma}(-z)=O\left(z^{-1}\right), \quad z \rightarrow+\infty .
$$

Then, using the representation

$$
\int_{0}^{\infty} \frac{e^{-a z}-e^{-b z}}{z} d z=\log (b)-\log (a), \quad \operatorname{Re}(a)>0, \operatorname{Re}(b)>0,
$$


we immediately get that

$$
\begin{aligned}
& \int_{0}^{\infty} z f(z) f(-z) e^{-\varepsilon z} d z \\
& =\sum_{l \in Q, k \in M_{\gamma}} \log \left(\lambda_{l}-i \xi_{k}+\varepsilon\right)-\sum_{l \in Q, m \in S} \log \left(\lambda_{l}+\mu_{m}+\varepsilon\right) \\
& +\sum_{j \in \overline{M_{\gamma}}, m \in S} \log \left(i \xi_{j}+\mu_{m}+\varepsilon\right)-\sum_{j \in \overline{M_{\gamma}}, k \in M_{\gamma}} \log \left(i \xi_{j}-i \xi_{k}+\varepsilon\right),
\end{aligned}
$$

where $\log (z)$ denotes the principal branch of the $\operatorname{logarithm}$, i.e., $\operatorname{Im}(\log (z))>0$ for $\operatorname{Im}(z)>0$. Taking the exponential function of both sides and passing to the limit as $\varepsilon \rightarrow 0$ we obtain the representation

$$
\begin{aligned}
& \exp \left(\lim _{\varepsilon \rightarrow+0} \int_{0}^{\infty} z f(z) f(-z) e^{-\varepsilon z} d z\right) \\
& =\frac{\prod_{l \in Q, k \in M_{\gamma}}\left(\lambda_{l}-i \xi_{k}\right) \prod_{j \in \overline{M_{\gamma}}, m \in S}\left(i \xi_{j}+\mu_{m}\right)}{\prod_{l \in Q, m \in S}\left(\lambda_{l}+\mu_{m}\right) \prod_{j \in \overline{M_{\gamma}}, k \in M_{\gamma}}\left(i \xi_{j}-i \xi_{k}\right)} .
\end{aligned}
$$

Comparing with Day's formula (1.6), we see that the r.h.s. of (2.24) obviously coincides with $W_{M_{\gamma}}$, which proves (2.22). Thus the proof of the theorem is completed.

\section{ACKNOWLEDGMENTS}

The financial support of SFB 237 given to the second author is gratefully acknowledged. This author also thanks the Mathematics Department of the Ruhr University Bochum for its kind hospitality.

\section{REFERENCES}

1. N. I. Ahiezer, The continuous analogue of some theorems on Toeplitz matrices, Ukrain. Mat. Zh. 16 (1964) 455-462; English transl., Amer. Math. Soc. Transl. (2) 50 (1966), 295-316. MR 30:411

2. S. Albeverio, R. Høegh-Krohn, and T. T. Wu, A class of exactly solvable three-body quantum mechanical problems and universal low energy behavior, Phys. Lett. A 83 (1981), 105-109. MR 82h:81017

3. S. Albeverio, S. Lakaev, and K. A. Makarov, The Efimov effect and an extended Szegö-Kac limit theorem, Lett. Math. Phys. 43 (1998), 73-85. MR 99e:47031

4. S. Albeverio and K. A. Makarov, Non trivial attractors in a model connected with the threebody Quantum Problem, Acta Applicandae Math. 48 (1997), 113-184. MR 99a:47033

5. S. Albeverio and K. A. Makarov, Limit behavior in a singular perturbation problem, regularized convolution operators and the three-body problem, Differential and Integral Operators (Regensburg, 1995), Operator Theory: Advances and Applications, vol. 102, Birkhäuser, Basel, 1998, pp. 1-10. MR 99i:81261

6. E. Basor, Asymptotic formulas for Toeplitz determinants, Trans. Amer. Math. Soc. 239 (1978), 33-65. MR 58:12484

7. A. Böttcher, Wiener-Hopf determinants with rational symbols, Math. Nachr. 144 (1989), 39-64. MR 91g:47017

8. A. Böttcher and B. Silbermann, The asymptotic behavior of Toeplitz determinants for generating functions with zeros of integer orders, Math. Nachr. 102 (1981), 79-105. MR 83f:47022

9. A. Böttcher and B. Silbermann, Wiener-Hopf determinants with symbols having zeros of analytic type, Seminar Analysis 1982/1983, 224-243, Akad. Wiss. DDR, Inst. Math., Berlin, 1983. MR 85e:47035]

10. A. Böttcher and B. Silbermann, Analysis of Toeplitz Operators, Springer-Verlag, Berlin, 1990. MR 92e:47001 
11. K. M. Day, Toeplitz matrices generated by the Laurent series expansion of an arbitrary rational function, Trans. Amer. Math. Soc. 206 (1975), 224-245. MR 52:708

12. M. E. Fisher and R. E. Hartwig, Toeplitz determinants: some applications, theorems, and conjectures. Adv. Chem. Phys. 15 (1968), 333-353.

13. M. E. Fisher and R. E. Hartwig, Asymptotic behavior of Toeplitz matrices and determinants, Arch. Rat. Mech. Anal. 32 (1969), 190-225. MR 38i:4888

14. L. D. Faddeev and S. P. Merkuriev, Quantum scattering theory for several particle systems. Kluwer, Dordrecht, 1993. MR 94j:81276

15. I. Gohberg, S. Goldberg, and M. A. Kaashoek, Classes of linear operators, Vol I. Operator Theory: Advances and Applications 49, Birkhäuser, 1990. MR 93d:47002

16. I. Gohberg and M. G. Krein, Introduction to the theory of linear nonselfadjoint operators in Hilbert space. Nauka, Moscow 1965 (Russian), Engl. trans.: Amer. Math. Soc. Transl. of Math. Monographs 18, Providence, R. I. 1969. MR 36:3137; MR 39:7447

17. I. Gohberg, M. A. Kaashoek, and F. van Schagen, Szego-Kac-Achiezer formulas in terms of realizations of the symbols, J. Func. Anal. 74 (1987), 24-51. MR 88m:47043

18. M. Kac, Toeplitz matrices, translation kernels and a related problem in probability theory, Duke Math. J. 21 (1954), 501-509. MR 16:31a

19. M. Kac, W. L. Murdock, and G. Szegö, On the eigenvalues of certain Hermitian forms, J. Rational Mechanics and Analysis 2 (1953), 767-800. MR 15:538b

20. H. Widom, Toeplitz determinants with singular generating functions, Amer. J. Math. 95 (1973), 333-383. MR 48:9441

Institut für Angewandte Mathematik, Universität Bonn, 53115 Bonn, Germany, SFB 237 (Essen-Bochum-Düsseldorf), BiBos (Bielefeld-Bochum/Bonn), CERFIM (Locarno)

E-mail address: Albeverio@uni-bonn.de

Department of Mathematics, University of Missouri, Columbia, Missouri 65211

E-mail address: makarov@azure.math.missouri.edu 